\title{
Efficacy and safety of sofosbuvir-based regimens for treatment in chronic hepatitis $C$ genotype 1 patients with moderately impaired renal function
}

\author{
Hyun Phil Shin', Ji-Ae Park', Blaire Burman², Richard A. Kozarek², and Asma Siddique ${ }^{2}$ \\ 'Division of Hepatology, Department of Internal Medicine, Kyung Hee University Hospital at Gangdong, Kyung Hee University School of \\ Medicine, Seoul, Korea, ${ }^{2}$ Department of Digestive Disease Institute, Virginia Mason Medical Center, Seattle, WA, USA
}

Background/Aims: Treatment of chronic hepatitis C virus (HCV) infection in patients with chronic kidney disease (CKD) is essential. The availability of sofosbuvir (SOF) has dramatically improved overall HCV cure rates, however there is insufficient data regarding its use in patients with CKD. We evaluated SOF in patients with hepatitis C genotype 1 (G1) and moderately impaired renal function.

Methods: We retrospectively reviewed all patients treated with a SOF-based regimen from December 2013 through September 2015 at Virginia Mason Medical Center. Data was then collected for HCV G1 patients with stage 3 CKD.

Results: A total of 28 patients with HCV G1 and stage 3 CKD were treated with a SOF-based regimen. Twenty-one patients had stage $3 A$ CKD (estimated glomerular filtration rate [eGFR] $45-60 \mathrm{~mL} / \mathrm{min} / 1.73 \mathrm{~m}^{2}$ ) and 7 patients had stage $3 B$ CKD (eGFR $30-45 \mathrm{~mL} / \mathrm{min} / 1.73 \mathrm{~m}^{2}$ ). The overall rate of sustained virologic response (SVR) 12 weeks after completion of therapy (SVR12) was $85.7 \%$ (24/28). SVR12 in stage 3A CKD patients was $81.0 \%(17 / 21)$ and in stage 3B CKD patients, SVR12 was $100 \%$ (7/7). Based on the treatment regimen used, the SVR12 was $81.8 \%(9 / 11), 92.3 \%(12 / 13)$, and $75.0 \%(3 / 4)$ for SOF/ledipasvir (LDV), SOF/simeprevir (SIM), and SOF/pegylated interferon (PEG)/ribavirin (RBV), respectively. Greater than $30 \%$ reduction eGFR was observed in 4 out of 28 patients.

Conclusions: SOF-based regimens resulted in high SVR12 rates in patients with moderately impaired renal function. During therapy, HCV patients with CKD should be carefully monitored for worsening renal function. (Clin Mol Hepatol 2017;23:316-322)

Keywords: Sofosbuvir; Chronic kidney diseases; Hepatitis C; Safety

\section{INTRODUCTION}

Hepatitis C virus (HCV) has infected over 170 million people worldwide ${ }^{1,2}$ and is one of the main causes of chronic liver disease in Korea. ${ }^{3} \mathrm{HCV}$ infection can cause renal disease in both native and transplanted kidneys including membranoproliferative glomerulone- phritis, with or without cryoglobulinemia, membranous nephropathy and significantly reduces graft survival in renal transplant recipients. ${ }^{4}$ It is reported that HCV patients have $23 \%$ greater relative risk of chronic kidney disease (CKD) compared to those without $\mathrm{HCV}^{5}$ It is therefore essential to treat HCV in patients with any stage of CKD.

\section{Abbreviations:}

CKD, chronic kidney disease; eGFR, estimated glomerular filtration rate; $\mathrm{HCV}$, hepatitis C virus; LDV, ledipasvir; PEG, pegylated interferon; SIM, simeprevir; SOF, sofosbuvir; SVR, sustained virologic response; RBV, ribavirin; RVR, rapid virologic response

\section{Corresponding author: Asma Siddique}

Digestive Disease Institute, Virginia Mason Medical Center, 1100 9th Avenue PO Box 900 Seattle, Washington 98101, USA

Tel: +1-206-223-2319, Fax: +1-206-223-6379

E-mail: Asma.Siddique@virginiamason.org

http://orcid.org/0000-0002-1284-6906 
Until recently the treatment of HCV infection in patients with CKD consisted of pegylated interferon (PEG) with or without ribavirin (RBV); however treatment outcomes were poor and side effects sometimes intolerable. ${ }^{6,7}$ With the availability of the direct acting antivirals (DAAs), substantial changes in the treatment of HCV in patients with CKD is anticipated. The American Association for the Study of Liver Diseases and the Infectious Diseases Society of America recommend that patients with mild-to-moderate renal impairment can be treated without any dose adjustment while using any of the recommended agents/regimens to treat HCV. In patients with severe renal impairment (estimated Glomerular Filtration Rate [eGFR] less than $30 \mathrm{~mL} / \mathrm{min}$ ), the FDA recommended regimen is standard-dose ombitasvir-paritaprevir-ritonavir and dasabuvir with or without ribavirin or fixed-dose elbasvir/grazoprevir. ${ }^{8}$

Sofosbuvir (SOF), a HCV NS5B nucleotide inhibitor, has primary renal clearance and therefore is not recommended in patients with estimated glomerular filtration rate (eGFR) less than $30 \mathrm{~mL} / \mathrm{min}$. A study in patients with advanced renal disease (eGFR<30 mL/ $\mathrm{min} / 1.73 \mathrm{~m}^{2}$ ) is currently in progress. SOF may be safe in treatment of HCV in patients with mild or moderate renal impairment, however there is extremely limited clinical data of its use in this unique patient population.

The aim of this current study is to define safety and efficacy of SOF-based regimens among those HCV-infected patients with moderate renal impairment in a real-world setting.

\section{PATIENTS AND METHODS}

\section{Patients}

We retrospectively reviewed electronic medical records of all adult patients with chronic HCV infection treated with SOF-based regimens at Virginia Mason Medical Center from December 2013 to September 2015. We then reviewed data of all HCV G1 patients treated with SOF-based regimens who had eGFR between 30-60 $\mathrm{mL} / \mathrm{min} / 1.73 \mathrm{~m}^{2}$ at baseline. eGFR was calculated by the modification of diet in renal disease study equation as $186 \times$ (Creatinine $/ 88.4)^{-1.154} \times(\text { Age })^{-0.203} \times(0.742$ if female $) \times(1.210$ if black $){ }^{9}$ eGFR $45-60 \mathrm{~mL} / \mathrm{min} / 1.73 \mathrm{~m}^{2}$ was defined as CKD stage $3 A$ and eGFR 30-45 mL/min $/ 1.73 \mathrm{~m}^{2}$ was defined as CKD stage 3B. The diagnosis of cirrhosis was made by liver biopsy and/or transient elastography with a median score above $12.5 \mathrm{kPa}$ being considered as consistent with cirrhosis (on a scale of 1.5 to $75.0 \mathrm{kPa}$ ). Treatment history was categorized into treatment naïve and treatment experi- enced based on history of previous HCV treatment.

Laboratory tests were checked prior to initiation of treatment, at least once a month throughout the treatment period, at the end of treatment, and post-treatment week 4 and week 12. Complete blood count, serum aminotransferase enzymes, albumin, total bilirubin, and serum creatinine were measured using standard laboratory procedures. Worsening of renal function was defined as a $30 \%$ reduction in eGFR from baseline at any point during treatment. ${ }^{10}$ HCV RNA levels were checked with a lower limit of quantification of $12 \mathrm{lU} / \mathrm{mL}$.

Definition of virologic response was undetectable HCV RNA levels. A rapid virologic response (RVR) was defined as undetectable HCV RNA at week 4 of treatment. We evaluated rate of sustained virologic response (SVR), as defined as undetectable HCV RNA 12 weeks post treatment (SVR12). Virologic relapse was defined as a serum HCV RNA level more than $12 \mathrm{IU} / \mathrm{mL}$ at anytime during treatment or during 12-week post-treatment period. HCV RNA level $>800,000 \mathrm{IU} / \mathrm{mL}$ was used as cut-off value high HCV RNA level. ${ }^{11}$ Adverse events on treatment were collected at clinic visits or by patient report throughout treatment period. Transfusion or use of erythropoietin during treatment was reviewed. This study was approved by the institutional review board of Virginia Mason Medical Center.

\section{Statistical analysis}

Categorical data are expressed as number (percentage), whereas continuous data are expressed as mean \pm SD. The Student's $t$ test was used to compare means, and the chi square test or Fisher's exact test was used to compare proportions. $P<0.05$ was considered statistically significant. Statistical analyses were performed using the Statistical Package for the Social Sciences (SPSS) version 18.0 for Windows (IBM Corp., Armonk, NY, USA).

\section{RESULTS}

\section{Baseline characteristics}

Twenty-eight patients with baseline CKD stage 3 were included after review of all HCV G1 patients treated with SOF-based regimen during this period. Majority of the patients had genotype $1 \mathrm{~A}$, and $50 \%$ of the patients had cirrhosis. Among the 28 patients, 11 patients were treated with SOF/LDV, 13 with SOF/simeprevir (SIM), and 4 with SOF/PEG/RBV. Although statistically not significant, the 
Table 1. Demographic and clinical characteristics of patients at baseline

\begin{tabular}{|c|c|c|c|c|}
\hline Baseline characteristic & CKD $3 A(n=21)$ & CKD 3B (n=7) & All patients $(n=28)$ & $P$-value \\
\hline Treatment regimen $(n, \%)$ & & & & $0.097^{*}$ \\
\hline SOF/LDV & $6(28.6)$ & $5(71.4)$ & $11(39.3)$ & \\
\hline SOF/SIM & $12(57.1)$ & $1(14.3)$ & $13(46.4)$ & \\
\hline SOF/PEG/RBV & $3(14.3)$ & $1(14.3)$ & $4(14.3)$ & \\
\hline Age (mean $\pm S D$, range), years & $61.0 \pm 11.2(27-78)$ & $62.9 \pm 5.4(56-72)$ & $61.4 \pm 10.0(27-78)$ & 0.67 \\
\hline Male Sex (n, \%) & $19(47.6)$ & $5(71.4)$ & $15(53.6)$ & $0.253^{*}$ \\
\hline Body-mass index (mean $\pm S D$, range) & $28.3 \pm 4.2(21-36.7)$ & $25.5 \pm 2.6(23.2-30.9)$ & $27.6 \pm 4.0(21-36.7)$ & 0.113 \\
\hline Race $(n, \%)$ & & & & $0.253^{*}$ \\
\hline White & $19(90.5)$ & $5(71.4)$ & $24(85.7)$ & \\
\hline African American & $1(4.8)$ & $1(14.3)$ & $2(7.1)$ & \\
\hline Other & $1(4.8)$ & $1(14.3)$ & $2(7.1)$ & \\
\hline HCV genotype $(n, \%)$ & & & & $0.674^{*}$ \\
\hline la & $14(66.7)$ & $4(57.1)$ & $18(64.3)$ & \\
\hline $1 b$ & $7(33.3)$ & $3(42.9)$ & $5(31)$ & \\
\hline Previous HCV treatment $(n, \%)$ & & & & $0.212^{*}$ \\
\hline Naïve & $12(57.1)$ & $5(72.4)$ & $17(60.7)$ & \\
\hline Treatment experienced (IFN based) $(n, \%)$ & $9(42.9)$ & $2(28.6)$ & $11(39.3)$ & \\
\hline Cirrhosis (n, \%) & $11(52.4)$ & $3(42.9)$ & $14(50.0)$ & $1^{*}$ \\
\hline Diabetes (n, \%) & $4(19.0)$ & $4(57.1)$ & $8(28.6)$ & $0.142^{*}$ \\
\hline \multicolumn{5}{|l|}{ Pre-laboratory test (mean $\pm S D$, range) } \\
\hline HCV RNA (log10 U/L) & $6.1 \pm 0.9(2.8-7.0)$ & $6.1 \pm 0.6(5.2-6.8)$ & $6.1 \pm 0.0(2.8-7.0)$ & 0.841 \\
\hline $\mathrm{Hb}(\mathrm{g} / \mathrm{dL})$ & $13.5 \pm 2.0(8.6-16.1)$ & $13.3 \pm 2.3(11.0-16.5)$ & $13.4 \pm 2.0(8.6-16.5)$ & 0.853 \\
\hline Platelet $\left(10^{9} / \mathrm{L}\right)$ & $144.1 \pm 72.5(29-284)$ & $153.4 \pm 44.0(85-214)$ & $146.5 \pm 65.9(29-284)$ & 0.754 \\
\hline PT (INR) & $1.14 \pm 0.16(0.9-1.4)$ & $1.04 \pm 0.09(1.0-1.2)$ & $1.12 \pm 0.03(0.9-1.4)$ & 0.195 \\
\hline Albumin (g/dL) & $3.7 \pm 0.6(2.0-4.6)$ & $3.7 \pm 0.4(3.0-4.2)$ & $3.7 \pm 0.6(2.0-4.6)$ & 0.91 \\
\hline $\mathrm{ALT}(\mathrm{U} / \mathrm{L})$ & $69.2 \pm 53.2(21-250)$ & $56.4 \pm 42.2(6-134)$ & $66 \pm 50.2(6-250)$ & 0.57 \\
\hline AST (U/L) & $62.5 \pm 32.8(25-160)$ & $47.3 \pm 27.2(13-87)$ & $58.7 \pm 31.7(13-160)$ & 0.279 \\
\hline Bilirubin (mg/dL) & $0.8 \pm 0.4(0.3-2.0)$ & $0.5 \pm 0.2(0.4-0.8)$ & $0.7 \pm 0.4(0.3-2.0)$ & 0.029 \\
\hline Creatinine (mg/dL) & $1.2 \pm 0.2(0.9-1.7)$ & $1.8 \pm 0.3(1.3-2.2)$ & $1.3 \pm 0.4(0.9-2.2)$ & $<0.001$ \\
\hline $\mathrm{CrCl}\left(\mathrm{mL} / \mathrm{min} / 1.73 \mathrm{~m}^{2}\right)$ & $55.2 \pm 3.7(47.4-59.9)$ & $36.6 \pm 6.4(30.0-44.2)$ & $50.6 \pm 9.3(30.0-59.9)$ & $<0.001$ \\
\hline
\end{tabular}

Values are presented as mean \pm SD or $n(\%)$ unless otherwise indicated. The body-mass index is the weight in kilograms divided by the square of height in meters.

CKD, chronic kidney disease; CKD 3A, estimated glomerular filtration rate (eGFR) 45-60 mL/min/1.73m²; CKD 3B, eGFR 30-45 mL/min/1.73m²; SOF, sofosbuvir; LDV, ledipasvir; SIM, simeprevir; PEG, pegylated interferon; RBV, ribavirin; SD, standard deviation; HCV, hepatitis C virus; IFN, interferon; $\mathrm{Hb}$, hemoglobin; PT, prothrombin time; INR, international normalized ratio; ALT, alanine transaminase; AST, aspartate transaminase; $\mathrm{CrCl}$, creatinine clearance.

${ }^{*}$ Fisher's exact test.

mean age was younger and the presence of cirrhosis was lower in the SOF/PEG/RBV group compared to the other 2 groups. According to baseline eGFR, 21 patients were regarded as having CKD stage $3 A$, and seven patients with CKD stage $3 B$. There were no statistically significant differences between CKD stage 3A and CKD stage 3B groups; with the exception of renal function and bilirubin level. Bilirubin level was higher in CKD 3A group, likely due to higher number of patients with cirrhosis in this group. Baseline characteristics are shown in Table 1. 




Figure 1. Virologic response by treatment regimen and baseline renal function. SVR, sustained virologic response; SOF, sofosbuvir; LDV, ledipasvir; SIM, simeprevir; PEG, pegylated interferon; RBV, ribavirin; CKD 3A, estimated glomerular filtration rate [eGFR] $45-60 \mathrm{~mL} / \mathrm{min} / 1.73 \mathrm{~m}^{2}$; CKD 3B, eGFR 30-45 mL/min/1.73m².

\section{Virologic response}

SVR12 was achieved in 24 of 28 patients (85.7\%). All 7 patients with CKD stage 3B achieved a SVR12. Among patients with CKD stage 3A, 81\% (17/21) achieved SVR12. Of the 4 patients with CKD stage $3 \mathrm{~A}$ who relapsed, 2 received SOF/LDV, 1 patient each received SOF/SIM and SOF/PEG/RBV. The treatment results of each regimen according to baseline renal function are provided in Figure 1.

SVR12 rates in patients who achieved RVR was higher than those who did not achieve RVR ( $90.0 \%$ vs. $60.0 \%, P=0.206)$ and SVR12 was higher in patients without cirrhosis compared to those with cirrhosis $(92.9 \%$ vs. $78.6 \%, P=0.302)$. SVR12 rates in patients less than 65 years old was higher compared to those 65 years or older $(94.4 \%$ vs. $70.0 \%, P=0.109)$. Based on genotype, SVR 12 was $94.4 \%(17 / 18)$ in patients with genotype $1 \mathrm{~A}$ and $70 \%(7 / 10)$ in patients with genotype $1 \mathrm{~B}(P=0.109)$. Statistically, there were no significant factors to predict treatment success. Treatment results according to population subgroup using univariate analysis are provided in Table 2.

\section{Adverse effects and safety}

All patients completed treatment, and only 1 patient failed to take RBV for 2 days. A full list of adverse events is provided in Table 3. Fatigue and headaches were the most common adverse events. No serious adverse events were reported. Four patients had worsening renal function, which included 2 out of 21 patients (9.5\%) with CKD stage $3 \mathrm{~A}$ and 2 out of 7 patients (28.6\%) with CKD stage 3B (Fig. 2). All 4 patients subsequently recovered renal function back to their baseline. Four patients had anemia defined as hemoglobin less than $10 \mathrm{~g} / \mathrm{dL}$ or over $2 \mathrm{~g} / \mathrm{dL}$ drop in hemoglobin. This included 3 patients (14.3\%) with CKD stage $3 A$ and 1 patient (14.3\%) with CKD stage 3B. In the CKD stage 3A group, 2 patients required
Table 2. SVR12 rates in patients by population subgroup

\begin{tabular}{|c|c|c|}
\hline Response & $\begin{array}{l}\text { SVR12 rates-no./ } \\
\text { total no. (\%) }\end{array}$ & $P$-value \\
\hline Chronic kidney disease stage & & $0.545^{*}$ \\
\hline $3 \mathrm{~A}$ & $17 / 21(81.0)$ & \\
\hline $3 B$ & $7 / 7(100)$ & \\
\hline Treatment regimen & & $0.469^{*}$ \\
\hline SOF/LDV & 9/11 (81.8) & \\
\hline SOF/SIM & $12 / 13(92.3)$ & \\
\hline SOF/PEG/RBV & $3 / 4(75.0)$ & \\
\hline Rapid virological response $^{\dagger}$ & & $0.166^{*}$ \\
\hline $\operatorname{RVR}(-)$ & $3 / 5(60.0)$ & \\
\hline $\operatorname{RVR}(+)$ & $18 / 20(90.0)$ & \\
\hline Cirrhosis & & $0.596^{*}$ \\
\hline Cirrhosis (-) & 13/14 (92.9) & \\
\hline Cirrhosis (+) & $11 / 14(78.6)$ & \\
\hline DM & & 0.555 \\
\hline DM (-) & $18 / 20(90.0)$ & \\
\hline $\mathrm{DM}(+)$ & $6 / 8(75.0)$ & \\
\hline Gender & & $1^{*}$ \\
\hline Male & $13 / 15(86.7)$ & \\
\hline Femele & $11 / 13(84.6)$ & \\
\hline Age & & $0.116^{*}$ \\
\hline$<65$ & 17/18 (94.4) & \\
\hline 65 & $7 / 10(70.0)$ & \\
\hline BMI & & $1^{*}$ \\
\hline$<30$ & $17 / 20(85.0)$ & \\
\hline 30 & $7 / 8(87.5)$ & \\
\hline Pretreatment HCV RNA (IU/mL) & & $1^{*}$ \\
\hline$<800,000$ & $6 / 7(85.7)$ & \\
\hline$\geq 800,000$ & $18 / 21(85.7)$ & \\
\hline Previous treatment & & $1^{*}$ \\
\hline Naïve & 15/17 (88.2) & \\
\hline Treatment experienced & 9/11 (81.8) & \\
\hline HCV genotype (n, \%) & & $0.116^{*}$ \\
\hline $1 \mathrm{a}$ & 17/18 (94.4) & \\
\hline $1 b$ & $7 / 10(70.0)$ & \\
\hline Race $(n, \%)$ & & $1^{*}$ \\
\hline Caucasian & 20/24 (83.3) & \\
\hline African American & $2 / 2(100)$ & \\
\hline Other & $2 / 2(100)$ & \\
\hline
\end{tabular}

The body-mass index is the weight in kilograms divided by the square of height in meters.

SVR12, sustained virological response 12 weeks post treatment; SOF, sofosbuvir; LDV, ledipasvir; SIM, simeprevir; PEG, pegylated interferon; RBV, ribavirin; RVR, rapid virological response; DM, diabetes mellitus; BMI, body mass index; HCV, hepatitis C virus.

"Fisher's exact test; ${ }^{\dagger}$ RVR had not been tested in three patients. 
Table 3. Adverse events by baseline renal function

\begin{tabular}{llll}
\hline Adverse events & CKD 3A $(\mathbf{n = 2 1 )}$ & CKD 3B $(\mathbf{n}=\mathbf{7})$ & All patients $(\mathbf{n}=\mathbf{2 8})$ \\
\hline Symptoms & 3 & 2 & 5 \\
\hline Fatigue & 2 & 1 & 3 \\
\hline Headache & 1 & 0 & 1 \\
\hline Abdominal discomfort & 1 & 0 & 1 \\
\hline Rash & 1 & 0 & 1 \\
\hline Dizziness & 1 & 0 & 1 \\
\hline Diarrhea & 1 & 0 & 1 \\
\hline Nausea & 1 & 0 & 1 \\
\hline Anxiety & 1 & 0 & 1 \\
\hline Eye discomfort & & & 2 \\
\hline Blood tests related & 1 & 1 & 2 \\
\hline Decreasing Hb level to $<10.0 \mathrm{~g} / \mathrm{dL}$ & 2 & 0 & 3 \\
\hline Decreasing Hb level to $<8.5 \mathrm{~g} / \mathrm{dL}$ & 2 & 1 & 2 \\
\hline Erythropoietin use during treatment. & 2 & 0 & 4 \\
\hline Transfusion for anemia & 2 & 2 & \\
\hline Worsening of renal function & 1 & \\
\hline
\end{tabular}

Worsening of renal function was defined as a 30\% reduction in eGFR from baseline during treatment.

CKD 3A, estimated glomerular filtration rate (eGFR) 45-60 mL $/ \mathrm{min} / 1.73 \mathrm{~m}^{2}$; CKD 3B, eGFR 30-45 mL/min/1.73m² Hb, hemoglobin.

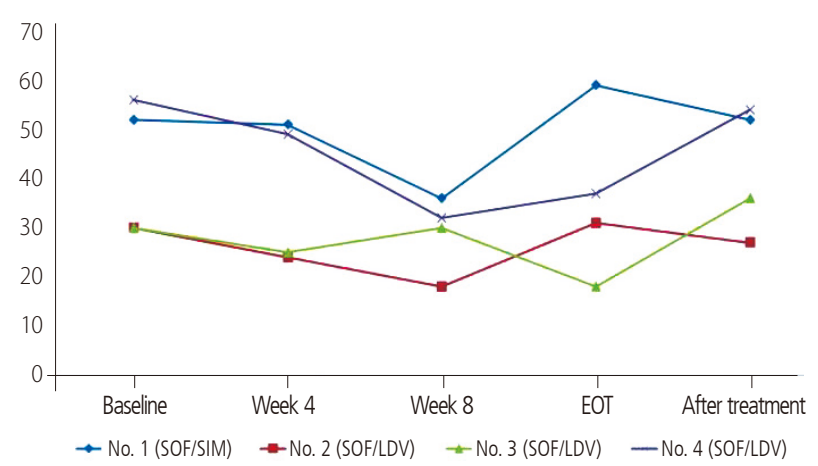

Figure 2. The change of estimated glomerular filtration rate (eGFR) in patients with worsening of renal function during treatment. EOT, end of treatment; SOF, sofosbuvir; SIM, simeprevir; LDV, ledipasvir.

transfusion, and 2 patients were treated with erythropoietin, while 1patient with CKD stage 3B required erythropoietin therapy.

\section{DISCUSSION}

SOF acts by inhibiting HCV NS5B RNA-dependent RNA polymerase, which is required for hepatitis $C$ viral replication. SOF is metabolized in the liver to its pharmacologically active metabolite (GS-461203), which gets incorporated into HCV RNA by the NS5B polymerase and acts as a chain terminator. It also produces inactive metabolite GS-331007, which enters the circulation and is renally eliminated. In patients with renal impairment, the area under the concentration-time curve (AUC) of GS-331007 is increased by $56 \%$ in mild, $90 \%$ in moderate and $456 \%$ in severe renal impairment subjects, compared to normal subjects. ${ }^{12}$ As a result SOF is not recommended in patient with severe renal impairment. Several clinical trials have evaluated the safety and efficacy of SOF with heterogenous results.

The HCV TARGET study, a multicenter prospective observation study, captured data from 1893 patients treated with a SOF based regimen. ${ }^{4} 168$ of these patients had baseline eGFR 46-60 mL/ $\mathrm{min} / 1.73 \mathrm{~m}^{2}, 55$ patients with eGFR $31-45 \mathrm{~mL} / \mathrm{min} / 1.73 \mathrm{~m}^{2}$ and 18 with eGFR $\leq 30 \mathrm{~mL} / \mathrm{min} / 1.73 \mathrm{~m}^{2}$, majority were genotype 1 . These patients were treated with SOF based regimen including SOF/PEG/ RBV, SOF/RBV or SOF/SIM with or without RBV. The overall SVR12 was $82-83 \%$ and was similar across eGFR groups. However, these patients experienced higher rates of worsening renal dysfunction and other serious adverse events. In comparison, other clinical trials did not report significant worsening renal function when treated with either full dose of SOF (400 mg) every other day or half dose (200 mg) daily, however these trials enrolled small number of patients. $^{12}$ 
Our study included regimens SOF/LDV, SOF/SIM and SOF/PEG/ RBV and is important because SOF/LDV is now available in genotype 1a and 1b with RAV in Korea although experience in CKD is insufficient yet. LDV has biliary clearance and therefore no dose reduction is required for patients with mild to moderate renal insufficiency. We specifically focused on patients with moderately impaired renal function.

In our study, the overall SVR12 rate in CKD stage 3 patients was high at $85.7 \%$. The SVR12 rate based on treatment regimen was $81.8 \%$ for SOF/LDV, $92.3 \%$ for SOF/SIM, and $75.0 \%$ for SOF/PEG/ RBV. Other studies with SOF based regimens have also shown high SVR rates and SOF has been found to be well tolerated both in moderate and severe renal impairment patients. ${ }^{13,14}$

Based on the CKD stage, SVR12 in patients with CKD stage 3A was $81.0 \%$ and in CKD stage 3B patients it was 100\%. Although there was no significant difference in baseline characteristics between the two groups, CKD 3A group had more treatment experienced patients (36.0\% vs. $28.6 \%$ ) and also higher number of patients with cirrhosis (52.4\% vs. $42.9 \%$ ) (Table 1).

In our study, 2 patients failed to achieve SVR in the SOF/LDV group. One patient was a 67-year-old Caucasian man, treatment naive with cirrhosis, and the second patient was a 78-year-old Caucasian woman, treatment experienced with stage 2 fibrosis. The patient in SOF/SIM group who failed to achieve SVR12 was a 70-year-old African-American man, treatment experienced with cirrhosis, and the patient treated with PEG/RBV/SOF that failed to achieve SVR was a 57-year-old Caucasian man with cirrhosis.

We observed worsening renal function to be more common in patients with CKD stage 3B (28.6\%) compared with CKD stage 3A $(9.5 \%)$. We performed a multivariate analysis to try and identify any factors that could predict worsening renal function in patients with CKD being treated with SOF based regimen, however no obvious risk factors were identified. This is maybe due to the fact that only a small number of patients experienced worsening renal function. Therefore renal function during SOF-based treatment should be carefully monitored in all patients with CKD stage 3 .

However, all patients recovered baseline renal function without discontinuation of treatment. In our study, of the 4 patients who experienced worsening renal function during therapy, 2 patients had compensated cirrhosis and were not on diuretics, and the other 2 patients were non-cirrhotics.

Anemia is a common adverse event in RBV including regimens, ${ }^{15}$ and CKD patients generally have a lower baseline hemoglobin level. Three patients (14.3\%) with CKD stage 3A and 1 patient (14.3\%) with CKD stage 3B had anemia with a hemoglobin level lower than
$10 \mathrm{~g} / \mathrm{dL}$ during treatment. Three of the patients received SOF/PEG/ RBV, and the fourth received SOF/SIM. Other adverse events were rare and not severe. Most common side effects were fatigue and headache. No patient discontinued treatment due to side effects.

We tried to analyze the predictors affecting treatment outcomes, but none were considered as predictors of SVR, possibly due to the small number of patients.

This study has unique strengths compared to that of previous studies. This is the first real-life treatment response and adverse effects data of CKD stage 3 patients using various SOF-based regimens in a single center. The limitations of this study are its retrospective nature and the small number of included patients. Moreover, the diagnosis of cirrhosis was made by different methods.

In conclusion, SOF-based regimens resulted in high SVR12 rates without serious adverse events in patients with moderately impaired renal function. Both CKD stage 3A and CKD stage 3B patients can be considered for treatment with a SOF-based regimen, however clinicians should be cautious and monitor for worsening of renal functions during treatment.

\section{Author contributions}

Asma Siddique and Hyun Phil Shin have full access to all of the data and have responsibility for the data and the data analysis.

- Study design: Asma Siddique, and Hyun Phil Shin

- Acquisition and interpretation of data: Asma Siddique, Blaire Burman, Hyun Phil Shin

- Drafting of the manuscript: Asma Siddique, Richard A. Kozarek

- Critical revision of the manuscript for important intellectual content: Asma Siddique, and Hyun Phil Shin

- Statistical analysis: Ji-Ae Park

\section{Acknowledgements}

This research was supported in part by The Research Fund of the Korean Association for the Study of the Liver (2015).

\section{Conflicts of Interest}

The authors have no conflicts to disclose.

\section{REFERENCES}

1. Ghany MG, Strader DB, Thomas DL, Seeff LB; American Association for the Study of Liver Diseases. Diagnosis, management, and treatment of hepatitis C: an update. Hepatology 2009;49:1335-1374. 
2. Shepard CW, Finelli L, Alter MJ. Global epidemiology of hepatitis C virus infection. Lancet Infect Dis 2005:5:558-567.

3. Shon HS, Choi HY, Kim JR, Ryu SY, Lee YJ, Lee MJ, et al. Comparison and analysis of the prevalence of hepatitis $C$ virus infection by region in the Republic of Korea during 2005-2012. Clin Mol Hepatol 2015;21:249-256.

4. Saxena V., Koraishy FM, Sise ME, Lim JK, Schmidt M, Chung RT, et al. Safety and efficacy of sofosbuvir-containing regimens in hepatitis Cinfected patients with impaired renal function. Liver Int 2016;36:807816.

5. Park H, Adeyemi A, Henry L, Stepanova M, Younossi Z. A meta-analytic assessment of the risk of chronic kidney disease in patients with chronic hepatitis C virus infection. J Viral Hepat 2015;22:897-905.

6. Berenguer M. Treatment of chronic hepatitis $\mathrm{C}$ in hemodialysis patients. Hepatology 2008;48:1690-1699.

7. Bunchorntavakul C, Maneerattanaporn M, Chavalitdhamrong D. Management of patients with hepatitis $C$ infection and renal disease. World J Hepatol 2015;7:213-225.

8. Smolders EJ, de Kanter CT, van Hoek B, Arends JE, Drenth JP, Burger DM. Pharmacokinetics, efficacy, and safety of hepatitis $C$ virus drugs in patients with liver and/or renal impairment. Drug Saf 2016;39:589-611.

9. Levey AS, Bosch JP, Lewis JB, Greene T, Rogers N, Roth D. A more accurate method to estimate glomerular filtration rate from serum creatinine: a new prediction equation. Ann Intern Med 1999;130:461-470.
10. Zeuzem S, Rodríguez-Torres M, Rajender Reddy K, Marcellin P, Diago $M$, Craxi $A$, et al Optimized threshold for serum HCV RNA to predict treatment outcomes in hepatitis $C$ patients receiving peginterferon alfa-2a/ribavirin. J Viral Hepat 2012;19:766-774.

11. Vardeny $\mathrm{O}, \mathrm{Wu}$ DH, Desai A, Rossignol P, Zannad F, Pitt B, et al. Influence of baseline and worsening renal function on efficacy of spironolactone in patients With severe heart failure: insights from RALES (Randomized Aldactone Evaluation Study). J Am Coll Cardiol 2012;60:2082-2089.

12. Cornprost M, Denning JM, Clemons D, Marbury TC, Alcorn H, Smith $W B$, et al. The effect of renal impairment and end stage renal disease on the single-dose pharmacokinetics of PSI-7977. J Hepatol 2012;56:5433.

13. Bhamidimarri KR, Czul F, Peyton A, Levy C, Hernandez M, Jeffers L, et al. Safety, efficacy and tolerability of half-dose sofosbuvir plus simeprevir in treatment of Hepatitis $C$ in patients with end stage renal disease. J Hepatol 2015;63:763-765.

14. Nazario HE, Ndungu M, Modi AA. Sofosbuvir and simeprevir in hepatitis $C$ genotype 1-patients with end-stage renal disease on haemodialysis or GFR < $30 \mathrm{ml} / \mathrm{min}$. Liver Int 2016;36:798-801.

15. Canonico PG, Kastello MD, Spears CT, Brown JR, Jackson EA, Jenkins DE. Effects of ribavirin on red blood cells. Toxicol Appl Pharmacol 1984;74:155-162. 